\title{
TOMOEDA Hiroyasu, El toro y el cóndor
}

Fondo Editorial del Congreso del Perú, Lima, 2013

Pablo F. Sendón

\section{(2) OpenEdition}

\section{Journals}

Edición electrónica

URL: https://journals.openedition.org/jsa/14005

DOI: 10.4000/jsa. 14005

ISSN: 1957-7842

Editor

Société des américanistes

\section{Edición impresa}

Fecha de publicación: 31 diciembre 2014

Paginación: 241-248

ISSN: 0037-9174

\section{Referencia electrónica}

Pablo F. Sendón, «tомоeda Hiroyasu, El toro y el cóndor», Journal de la Société des américanistes [En línea], 100-2 | 2014, Publicado el 01 julio 2015, consultado el 02 septiembre 2022. URL: http:// journals.openedition.org/jsa/14005 ; DOl: https://doi.org/10.4000/jsa.14005

Este documento fue generado automáticamente el 2 septiembre 2022.

All rights reserved 


\title{
TOMOEDA Hiroyasu, El toro y el cóndor
}

\author{
Fondo Editorial del Congreso del Perú, Lima, 2013
}

\author{
Pablo F. Sendón
}

\section{REFERENCIA}

TOMOEDA Hiroyasu, El toro y el cóndor, Fondo Editorial del Congreso del Perú, Lima, 2013, 353 p., bibl., photos, cartes

1 La traducción al castellano de una etnografía escrita en japonés dedicada a las poblaciones campesino-indígenas de los Andes no sólo resulta un suceso editorial sino que también despierta el interés - o debería hacerlo - de los especialistas que, desde diversos rincones del mundo, se ocupan de temáticas y problemáticas afines. Incluso más, debido a la impericia de la gran mayoría de los especialistas para leer el idioma en el que el volumen por comentar fue concebido, las casi tres décadas que transcurrieron desde su publicación original (1986) no deberían ser motivo para desestimar el hecho de que, planteado en estos términos, nos encontramos ante una " primera edición » ${ }^{1}$.

2 Las investigaciones arqueológicas y antropológicas japonesas en los Andes datan de 1959, año del inicio de lo que se denominó la «Expedición científica de Japón en América nuclear " patrocinada por la Universidad de Tokio y a la que, junto con un grupo de colegas japoneses y peruanos, se incorporó en 1963 Hiroyasu Tomoeda en calidad de fotógrafo documentalista. Desde entonces y hasta su muerte (2009) Tomoeda realizó investigaciones etnológicas en la sierra central y meridional del Perú, el alto Amazonas, Bolivia y el norte peruano. Junto con su labor etnológica, que se tradujo en numerosos libros y artículos, Tomoeda dejó un legado de más de 45 mil fotografías tomadas mediante diversos dispositivos durante los poco más de 45 años que dedicó a los Andes y que, donación de por medio, desde 2005 son conservadas en el museo de Antropología de la Universidad de Nanzan. El libro que comentamos, resultado de su tesis de magíster escrita en 1981, pertenece a lo que el mismo Tomoeda consideró como la «etapa formativa de la etnología andina en el Japón » (p. 24)². 
3 Anclado en diversas poblaciones campesino-indígenas y quechua hablantes del la provincia de Aymaraes (departamento de Apurímac), y en contrapunto con la información procedente de otras regiones de los Andes peruanos e incluso bolivianos, El toro y el cóndor es una etnografía que despliega, de manera paulatina y sutil, una serie de contrastes en su mutua relación. No es de extrañar, entonces, que el escenario que da inicio al primer capítulo dedicado al mundo metafórico de los pastores de puna (localizados por encima de los 4,000 metros sobre el nivel del mar) sea la costa del océano Pacífico. En efecto, los pastores apurimeños realizan anualmente viajes con sus llamas hacia las playas del Pacífico para aprovisionarse de algas marinas (cocha yuyo) con el objeto de intercambiarlas por maíz en los valles quichua (entre los 2,300 y 2,500 metros de altitud) así como por coca en las yungas de las pendientes occidentales y orientales de las cordilleras (entre los 500 y 2,000 metros de altura) constituyéndose, de este modo, en lo que se ha denominado verdaderos « tejedores de espacio " ${ }^{3}$. A pesar de su alta movilidad, que supo relacionarlos con ecologías y sociedades diversas, los pastores como tales detentan una identidad propia (asociada con el término punaruna) que contrasta, en particular, con la de los agricultores (quichuaruna) que habitan en pisos ecológicos adyacentes. Los hábitos y modos de vida de ambos tipos de sociedades hacen de ellas " grupos sociales cerrados entre sí » (p. 69), reservándose los calificativos de uywa michiq (pastor) o sallqakuna (gente que vive en territorios inhóspitos) para los primeros, y chakrayuq (persona que tiene chacra) y ripuqtaña (gente que vive en el pueblo) para los segundos. Esto tiene un correlato en la excepcional tasa de matrimonios celebrados entre pastores y agricultores a lo largo del tiempo. Sin embargo, entre uno y otro grupo existen relaciones económicas complementarias que los comprometen en un profuso intercambio de bienes y servicios a lo largo del ciclo anual. Esta complementariedad tiene, a su vez, un correlato en los ritos propiciatorios dedicados a la fertilidad de los animales domésticos celebrados en el mes de agosto (agustukuy, ayllusqa o llama tinka). Esto se aprecia, significativamente, en las canciones dedicadas a las llamas y a las alpacas durante el tiempo ritual, en las expresiones que se emplean para dirigirse a ellas y en los tratamientos a los que son sometidas. En las primeras, el deseo de reproducción de los animales es expresado metafóricamente mediante el deseo de reproducción de la flora. En las segundas, encontramos que para el pastor « la llama es mi chacra » (p. 91), el acto de trasquilar la lana de la alpaca se denomina « cosecha lana» (p. 92), los viajes a los valles con recuas de llamas para realizar el trueque son llamados « intercambiar mi maíz blanco con el tuyo » (ibid.), las acciones de distribuir la carne (mastay mallichiy) y recibir productos agrícolas (wantuy) durante el intercambio son referidas como "sembrar » y " cosechar» (ibid.) y que el maíz molido (llampu) y la hoja de coca simbolizan el « cuerpo » y el « alma » (p. 93) de los animales. Entre los terceros destaca el empleo de la huaylla (clase de ichu que crece en la puna donde hay agua de montaña) para adornar a los animales durante la tinka: sobre sus lomos se disponen tres pastos a la manera de líneas ramificadas que, en virtud del empleo del verbo huayllariy, permite concebir la reproducción del ganado en términos « ramificar como huaylla » (p. 95).

El segundo capítulo nos traslada a un piso altitudinal inmediatamente inferior (entre las zonas puna y suri, alrededor de los 3,400 metros) apto para la crianza de ganado europeo a propósito de la fiesta de marcación dedicada a los toros y a las vacas (herranza o qerakuy) celebrada en el mes de abril (día de San Marcos). El objeto del análisis consiste no sólo en subrayar la presencia de elementos similares en los rituales dedicados a los animales domésticos precolombinos (señalakuy) - en particular el empleo metafórico del mundo vegetal - sino en estudiar lo que el autor denomina la «ideología de la fuerza de 
reproducción mística » (p. 138) en virtud de la cual « el rito de los animales en los Andes lleva la fuerza de reproducción mística al mundo real de los humanos donde se hace posible su utilización» (p. 138). En los términos que propone el plano de la acción, esta ideología se expresa en un rito de transición que contempla las etapas de la víspera, la herranza (o marcación) propiamente dicha y el entierro de la señal. Veamos. La fuerza de reproducción de los animales domésticos está asociada a una fuerza subterránea caracterizada con los nombres de Pachamama o Taitaorqo. En el ámbito del rito, el mundo subterráneo está simbolizado en el mesa qepi, altar de tela donde se depositan objetos diversos entre los que sobresalen las illa, pequeñas figuras zoomorfas de piedra, a través de las cuales se expresa la fuerza (la reproducción) del mundo subterráneo. El tratamiento que reciben estas figuras (conservadas celosamente por los campesinos) a lo largo del rito - consistente sobre todo en libaciones de licor (tinka) sobre y alrededor de ellas - informa que su propia existencia y, a través de ellas, la del propio mundo místico es favorecida por los participantes del ritual promoviendo entre un mundo y el otro, un estado de equilibrio en virtud del cual se asegura la reproducción de animales (domésticos) y humanos. Las diferentes ofrendas realizadas al mundo místico (orejas, rabos, sangre y grasa de los animales así como comida y licor) advierten la existencia de un estado de intercambio constante entre ambos mundos (incluso de devolución de uno hacia el otro) que se traduce en un contraste entre la síntesis (la fuerza sintética del mundo místico) y la diseminación (la dispersión o la ramificación de los animales en este mundo) involucrando también, el segundo de los términos, la idea misma de decaimiento y muerte de la fuerza de reproducción. De aquí la necesidad de reunir a los animales domésticos en el corral una vez al año para marcarlos o señalarlos.

El tercer capítulo nos ubica en la zona quichua (entre los 2,300 y 3,400 metros de altura) en la que se combina el cultivo de maíz en la parte baja y el de papa en la parte alta. El motivo de este nuevo descenso es presentar y analizar las fiestas de la corrida de toros y el cóndor rachi en el pueblo de Caraybamba (Apurímac) desde la perspectiva que ofrecen los ámbitos de la organización social y el parentesco. Aunque de origen foráneo y relacionadas en términos generales con hitos del calendario litúrgico cristiano (en particular la fiesta patronal de la Virgen del Rosario), estas fiestas parecen adecuarse a pautas de organización y relaciones de parentesco típicamente "andinas" o, si se prefiere, a través de las primeras las segundas se expresan de manera elocuente. En la corrida de toros de corte tradicional, se elige un encargado (carguyoq) para la obtención de toros bravos de cada uno de los barrios en que se divide el pueblo: Hanan barrio y Uray barrio. El primer día de la corrida está a cargo del carguyoq del barrio de arriba y el segundo del carguyoq del barrio de abajo. La bipartición del pueblo está asociada con su propia geografía, ya que cada barrio se ubica en las márgenes izquierda y derecha de la quebrada que forman los cerros Apumarca y Piste, ambos considerados apus tutelares de los ganados. El carguyoq del Hanan barrio ofrece entonces los toros criados en la margen izquierda del pueblo que, en su representación, compiten con los toros de la mitad opuesta en bravía y destreza. El cóndor rachi, más célebremente conocido como yawar fiesta a partir de la pluma de José María Arguedas, es una variación de la corrida tradicional consistente en atar un cóndor vivo con una pita de cuero sobre el lomo del animal por torear. La descripción del evento, si bien escalofriante, no es menos ilustrativa. El cóndor (concebido también como la gallina, es decir el animal doméstico, del $a p u$ ) y el toro están íntimamente ligados al espíritu de los cerros y los términos que los oponen son los mismos que contrastan los universos de lo salvaje y lo doméstico, el cielo y la tierra, el carnívoro y el herbívoro: el cóndor baja desde el cielo en busca de 
carroña y el toro mágico (illa) aparece desde las entrañas de las lagunas en busca de vacas con las que copular insaciablemente. El encuentro de ambos animales en la plaza de la corrida, en lugar de representar los opuestos de un antagonismo insalvable, evoca el antagonismo en sí mismo en su función generadora. En este sentido, el cóndor rachi es interpretado por Tomoeda como una variación morfológica de los rituales propiciatorios del ganado. Pero esto no es todo. En la esfera de las relaciones sociales, el toro representa a un «nosotros» en contraposición al cóndor que representa a los «ajenos». Ambas instancias se encuentran corporizadas, respectivamente, en los roles del carguyoq y el de su « hijo político », quien en el ámbito del rito desempeña labores secundarias, más no por ello menos indispensables, tales como administrar, observando las maneras de la mesa (hay que tener en mente que en la mitología andina el cóndor es descrito muchas veces como un comensal educado y elegante, vestido de obligada etiqueta negra adornada con una chalina blanca), los trozos descuartizados del toro ofrecido en sacrificio o dirigir el juego del toro queso, consistente en desatar y distribuir tres quesos que penden de unas soguillas (wallqapas) adornados con las mismas cintas de colores con las que se señala a los animales en los ritos propiciatorios. Ahora bien, lo que hasta aquí se ha identificado como " hijo político " no son sino los hombres agrupados bajo los términos de parentesco masa o qatay ( $\mathrm{ZH}$ o $\mathrm{DH}$ ) que detentan una notable extensión clasificatoria de la cual Tomoeda es bien consciente. El masa (o qatay en el sur peruano) es el hombre perteneciente a un ayllu que no es el suyo en virtud del vínculo matrimonial celebrado con una de sus mujeres. De ahí su condición de " ajeno ", que es acentuada aún más en el rito del matrimonio a propósito del cual el masa es considerado como un ser (un cóndor) que arrebata y roba a las mujeres del ayllu. Más allá de que la definición y caracterización del ayllu propuesta por Tomoeda presenta una serie de dificultades que exceden el ámbito de estas líneas, lo cierto es que sus reflexiones sobre la condición de masa en calidad de « lo ajeno " presentan una serie de matices que se acercan a - o mejor aún, anuncian - conclusiones similares surgidas de estudios recientes sobre parentesco en los Andes relativas a la misma cuestión: « Así, dos lugares que ocupa un individuo en la relación de parentesco, o sea, pertenecer a su propio aillu y ser 'ajeno' en otro aillu, son paralelos y equilibrados. En la función cotidiana, como colaboración laboral o préstamos económicos, no hay mucha distinción entre la familia consanguínea y la familia formada por el matrimonio, sin dar mucha evidencia de la condición ajena de un individuo en las escenas de la coordinación y la colaboración»(p.246). Parecería entonces que la condición « ajena » del masa estaría reservada a (o sería acentuada en) la esfera ritual. Sin embargo, en este punto de su razonamiento Tomoeda no deja de advertir que « la unidad básica de la organización social de los Andes es el individuo que representa al aillu y su masa, o sea, sin masa no se realiza el ritual » (p. 251). A esto mismo nos referíamos líneas arriba cuando observábamos (cosa que no hace Tomoeda) la función generadora del antagonismo expresado en la unión del toro y el cóndor durante la corrida: sin masa no se realiza el ritual y sin masa, nos permitimos agregar, no se realiza el ayllu ${ }^{4}$.

6 El último capítulo del libro involucra un cambio de escala y un último descenso. El objetivo aquí es estudiar los mitos (del zorro y del chihuaco) y ritos (cortamonte y juegos infantiles) relativos, de manera más o menos explícita, al origen de las plantas cultivadas procedentes de todos los Andes peruanos y el norte boliviano desde la perspectiva que ofrecen sus contrapartes de la alta Amazonía o, en términos más precisos, « hacer que los mitos amazónicos interpreten a los cuentos andinos» (p. 264)5. Es imposible resumir la riqueza del análisis al que fueron sometidos decenas de cuentos andinos desde la 
perspectiva adoptada, por lo que nos circunscribiremos a sus lineamientos generales y a unos cuantos ejemplos.

7 En muchas de sus variaciones, el cuento del zorro (pícaro, mentiroso y glotón) relata su ascenso a un banquete celestial con la ayuda de un cóndor. Tras comer en exceso y no haber observado las reglas de etiqueta, el primero fue abandonado por su acompañante. Deseoso de regresar a la tierra, el zorro construyó una soga y, en su descenso, insultó a una banda de loros que cruzaban el cielo. Éstos, indignados, cortaron la cuerda, el zorro se estrelló sobre la tierra y de su vientre repleto de alimentos surgieron las plantas cultivables. En una de las versiones andinas del cuento, el protagonista es un joven que se comporta de la misma manera que el zorro en su larga estancia en la casa de una doncella celestial. Al ser abandonado por ella, el cóndor ayuda al joven en su descenso y, bañándose juntos en el mar del cielo, rejuvenecen antes de su retorno a la tierra. En la mitología machiguenga se encuentran los motivos del origen de las plantas cultivadas y el de la soga que une a la tierra y el cielo, mientras que, por su parte, un mito piro sobre la vida eterna relata, de manera inversa al segundo de los cuentos andinos, el motivo de por qué los hombres no consiguieron el rejuvenecimiento en una época posterior a la del origen de las plantas cultivadas. Los motivos comunes en la saga del zorro son la disyunción arriba-abajo y el desplazamiento-dispersión del objeto precipitado. Este segundo motivo se repite en el cortamonte de carnaval. Este rito consiste en hacer un hueco en la tierra en el que se coloca el tronco de un árbol alto y pesado, especialmente engalanado para la ocasión con dones de todo tipo, con el propósito de derribarlo y acceder a ellos tras su caída estrepitosa. En la mitología cashinahua existe el motivo de un árbol gigante que sostiene y separa la tierra del cielo y que los seres míticos tratan de derribar infructuosamente. Un modelo similar (y para Tomoeda incluso precedente) al cortamonte andino se aprecia en el rito huitoto del « jalico » o yadic, el cual se celebra meses después de la preparación de la quema y la roza, y que también dramatiza el origen de las plantas cultivadas tras el derrumbe de un árbol pesado. Los cuentos del chihuaco relatan que Dios (o el Padre Sol) encomendó a esta ave trasmitir a los hombres su deseo de que vivan frugalmente, pero el chihuaco hizo lo contrario y les dijo a los hombres que cultiven plantas y críen animales, que cocinen sus alimentos y que coman hasta tres veces por día, propiciando de este modo la decadencia del hombre en su existencia terrenal. Aunque expresados de manera tenue, los cuentos del chihuaco remiten a los mitos amazónicos que relatan el origen del envejecimiento y la muerte como resultado o compensación del origen de las plantas cultivables y el fuego. Todos estos motivos aparecen en los juegos infantiles registrados en varios sitios de los Andes en los que un niño representa un árbol que debe ser tumbado por sus compañeros.

8 A propósito justamente del ejercicio de demarcación de los territorios de Sudamérica en "áreas culturales» realizado por una tradición del pensamiento antropológico, Tomoeda sugiere que «sería útil para estudios futuros tratar en algunos casos ambos territorios [Andes y Amazonía] como un solo grupo, sobre todo las áreas donde desde tiempo remoto mantienen una continua interacción» (p. 264). En lo que respecta a los cuentos, mitos, juegos y ritos analizados, nuestro autor concluye que todos ellos « forman un conjunto significativo que deviene del pensamiento del hombre andino y que este conjunto es parte del sistema total en que los mitos amazónicos también se encuentran integrados » (p. 265).

Dijimos al comienzo que esta etnografía despliega una serie de contrastes en su mutua relación. Desde las costas del Pacífico hasta el interior de la Amazonía - pasando por 
todos los pisos altitudinales posibles -, y desde los rituales consagrados a las llamas y a las alpacas hasta las corridas de toros de tradición ibérica y la mitología de las « tierras bajas ", El toro y el cóndor ofrece una visión de conjunto de la vida de las poblaciones campesino-indígenas de los Andes sin perder de vista sus más íntimos detalles. En lo que a nosotros respecta, no podemos sino recomendar al lector de estas líneas deshacer el camino recorrido y observar los diversos pliegues abiertos hasta aquí en su intrincada combinación y armonía y así apreciar, a la manera de una pieza de origami, el resultado de la obra reseñada en toda su completitud, áreas y volúmenes.

\section{BIBLIOGRAFÍA}

\section{HARRIS Olivia}

2008 « Alterities: kinship and gender in Latin American anthropology », in D. Poole (ed.),

A companion to Latin American anthropology, Blackwell, Malden, pp. 276-302.

\section{KIMURA Hideo}

1985 « Andean exchange: a view from Amazonia », in S. Masuda, I. Shimada y C. Morris (eds), Andean ecology and civilization: an interdisciplinary perspective on Andean ecological complementarity, University of Tokyo Press, Tokio, pp. 491-504.

MASUDA Shozo (ed.)

1981 Estudios etnográficos del Perú meridional, Universidad de Tokio, Tokio.

MASUDA Shozo, Izumi SHIMADA y Craig MORRIS (eds)

1985 Andean ecology and civilization: an interdisciplinary perspective on Andean ecological complementarity, University of Tokyo Press, Tokio.

RICARD LANATA Xavier y Gustavo VALDIVIA CORRALES

2009 Tejedores de espacio en los Andes. Itinerarios agropastoriles e integración regional en el sur peruano, Centro de estudios regionales andinos Bartolomé de Las Casas/Grupo voluntariado civil de Italia, Cuzco.

SENDÓN Pablo F.

2006 « Los términos de parentesco quechua qatay y qhachun según los registros etnohistóricos y etnográficos: una interpretación », Revista andina, 43, pp. 9-58.

\section{TOMOEDA Hiroyasu}

1988 " "La llama es mi chacra": el mundo metafórico del pastor andino », in J. A. Flores Ochoa (ed.), Llamichos y paqocheros. Pastores de llamas y alpacas, Consejo nacional de ciencia y tecnología/Centro de estudios andinos, Cuzco, pp. 225-235.

\section{NOTAS}

1. Lo decimos al comienzo para no desvirtuar los párrafos que siguen: es imperdonable que la presente traducción no haya recibido un trabajo serio de corrección de estilo ya que, tal y como se ha presentado, la lectura del texto resulta en muchos momentos áspera, por decir lo mínimo. 
2. Parte de los resultados del conjunto de investigaciones realizadas durante esta primera etapa se dieron a conocer en dos volúmenes publicados tempranamente en castellano y en inglés: Masuda (1981) y Masuda et al. (1985). La presente edición cuenta con un listado confeccionado por el antropólogo peruano Luis Millones de las publicaciones de Tomoeda disponibles en español. En la lista se ha omitido un título significativo ya que corresponde a una síntesis apretada del primer capítulo de la etnografía que realizara su autor para una obra colectiva dedicada al estudio de sociedades de pastores en los Andes (Tomoeda 1988).

3. Ver Ricard Lanata y Valdivia Corrales (2009).

4. Para un análisis sobre la condición « relativa " del masa o qatay en la estructura del ayllu contemporáneo, y del afín en general en el ámbito de las sociedades «andinas » y « amazónicas » ver, respectivamente, Sendón (2006) y Harris (2008).

5. Si bien existen antecedentes meritorios en este tipo de empresa relativa a cuestionar los supuestos límites que separan los universos de las «tierras altas » y las «tierras bajas » de esta porción del continente, en este contexto es digno de recordar un artículo de Hideo Kimura (1985), colega de Tomoeda, también perteneciente a la etapa formativa de la etnología andina en el Japón.

\section{AUTORES}

PABLO F. SENDÓN

CONICET, Argentina 